\title{
KANT AND THE IMPOSSIBILITY OF NON-EUCLIDEAN SPACE
}

\section{TUFAN KIYMAZ}

In this paper, I discuss the problem raised by the non-Euclidean geometries for the Kantian claim that the axioms of Euclidean geometry are synthetic a priori, and hence necessarily true. I argue that, although the Kantian view of geometry faces a serious challenge from non-Euclidean geometries, there are some aspects of Kant's view about geometry that can still be plausible. However, I will not reduce synthetic a priori Euclidean geometry to simply what Strawson ${ }^{1}$ calls "phenomenal geometry," where "phenomenal," in this context for Strawson, loosely means "visualizable" or "picturable." Phenomenal geometry, in this sense, is the geometry of imagined scenes. If Euclidean geometry is only necessarily true for imagined/ visualized objects and their imagined spatial relations, but not for empirical/ sensible world, then I take it that Kant was completely wrong about the nature of geometry. In what follows, I try to save some of Kant's views, without separating geometry from the empirical world and confining it solely into mental imagery.

Necessity of the fundamental laws that govern appearances (phenomena, in the Kantian sense) is central to Kant's understanding of the notion of "natural science." According to Kant, these fundamental laws can be necessary only if they are cognized a priori. He writes, in Metaphysical Foundations of Natural Science:

A rational doctrine of nature thus deserves the name of a natural science, only in case the fundamental natural laws therein are cognized a priori, and are not mere laws of experience ${ }^{2}(468)$

${ }^{1}$ P. F. Strawson, The Bounds of Sense: An Essay on Kant's Critique of Pure Reason (London: Methuen, 1966).

2 Immanuel Kant, Metaphysical Foundations of Natural Science. ed. by Michael Friedman (Cambridge: Cambridge UP, 2004). 
Kant maintains that if the fundamental principles of a systematic doctrine about a particular subject depend on empirical facts rather than a priori faculties, those principles can only belong to a systematic art, but not to a proper natural science. Kant takes geometry as a natural science with necessary fundamental principles (axioms) whose subject matter is space.

According to Kant, our a priori (pure) intuitions determine the form of our sensibility and hence the sensible world that we live in. All appearances exist in space and time. Kant believes that Euclidean geometry is necessary because it is grounded on our a priori (and hence necessary) intuition of space and our intuition of space necessitates the truth of the axioms of Euclidean geometry. So, a priori intuitions have two distinct roles: they are the grounds of the possibility of the sensible world, and also they are the grounds of the possibility of our knowledge (sciences) of the sensible world. Particularly, intuition of space is, for Kant, both a basis for the Euclidean nature of the appearances and their spatial relations in the sensible world and also a basis for Euclidean geometry as an exact natural science.

I will call the world of appearances, the physical world that we live in the "sensible world," as Kant does too, and the theoretical world that is depicted by our scientific theories the "scientific world." Of course the subject matter of sciences like physics and geometry is the sensible world, but in order to explain the sensible world, they propose a theoretical world. For example, Newtonian physics and Einsteinian physics give us two different scientific worlds which are supposed to represent the sensible world.

Now, we can turn to the challenges posed by the non-Euclidean geometries to Kant. Non-Euclidean geometries are consistent formal systems and they deny the 5th Euclidean axiom (the parallel axiom) which Kant takes to be necessarily true. One line of argument is that the existence of non-Euclidean geometries show that the axioms of Euclidean geometry are not necessarily true, and hence not a priori ${ }^{3}$.

One of the standard arguments against the Kantian theory of geometry can be phrased as follows:

1. If the 5th axiom is a priori, then it is not possibly false.

2. If there are consistent geometries in which the 5th axiom is false, then the 5 th axiom is possibly false.

3. There are non-Euclidean geometries and they are consistent geometries in which 5th axiom is false.

4. Therefore, the 5th axiom is not a priori.

\footnotetext{
3 Jerrold Katz, for example, writes: "Einstein's relativistic physics refuted Kant's claim that Euclidean geometry expresses synthetic a priori knowledge of space, thereby not only depriving Kant of an account of geometrical knowledge, but also, and more importantly, putting his entire account of synthetic a priori knowledge under a cloud of suspicion" (292) in Jerrold Katz, The Metaphysics of Meaning (Cambridge, MA: MIT Press, 1990).
} 
Let us call this the "possibility argument." The argument could be phrased in terms of conceivability and not possibility, but this formulation will help us distinguish between the epistemology of Euclidean geometry and the metaphysics of Euclidean space, which, as I will argue, is a crucial distinction. I will consider a version of the argument that rests solely on conceivability of non-Euclidean geometries, which was proposed by Poincare, below.

The possibility argument is valid. However, premise 2 is questionable. Does the fact that there are consistent non-Euclidean geometries imply that the 5th axiom is possibly false?

I do not think that the fact that we can construct a geometry as a coherent formal system when we deny Euclid's 5th axiom shows that Euclid's 5th axiom is not necessarily true. For example, there are some logical systems in which the law of non-contradiction does not hold. For logical systems like dialetheic paraconsistent logic "p and not p" can be true. But, I do not think it is obvious that just because there are some deviant logics (as Quine calls them) the law of non-contradiction of classical logic is not a necessary truth. Another, and a less controversial, example can be the negative numbers. Negative numbers are a part of the mathematical system we use, however, just because we use them to explain quantitative relations among objects, say apples, we cannot say that -1 apple is possible in reality like 1 apple. Similarly, it can be claimed that the mere existence of non-Euclidean geometries does not show that the sensible world is possibly non-Euclidean. In other words, logical possibility may not entail metaphysical possibility.

However, even though premise 2 is not plausible, we seem to have other reasons to believe that the sensible world is not necessarily Euclidean. Non-Euclidean geometries are utilized in the general theory of relativity, which is currently our best scientific theory concerning space (or, to be more precise, space-time). So, to amend the above formulation of the possibility argument, we can modify premise 2 as follows:

$2^{\prime}$. If there are consistent geometries in which the 5 th axiom is false and we use at least one of them in our best scientific theory concerning space, then the 5th axiom is possibly false.

If we revise premise 3 accordingly, as follows, we have again a valid argument.

$3^{\prime}$. There are non-Euclidean geometries and they are consistent geometries in which 5th axiom is false and we use at least one of them in our best scientific theory concerning space.

But, is $2^{\prime}$ true? $2^{\prime}$ depends on the idea that if $\mathrm{P}$ is true in the scientific world that our best theory describes (in order to explain the sensible world), then $\mathrm{P}$ is possibly true in the sensible world. A stronger claim would be that $\mathrm{P}$ is not only possibly but probably, or even actually, true in the sensible world, but I will just consider the weaker claim because the mere possibility of the falsity of Euclid's axioms is sufficient to show that Kant's theory about the Euclidean space is false. 
One, however, might try to show that the sensible world cannot be nonEuclidean by claiming that the sensible world's being non-Euclidean is in fact inconceivable (and hence, according to Kant, metaphysically impossible), and if the sensible world cannot be non-Euclidean, then $2^{\prime}$ is false. But then we have to explain the nature of the very precise correspondence between the relativistic scientific world and the sensible world, especially concerning very fast and very massive objects. First, let us look at the claim that a non-Euclidean world is inconceivable and hence impossible.

Strawson states that it is "evident that we cannot either in the imagination or on paper, give ourselves a picture such that we are prepared to say of it both that it shows two distinct straight lines, and that it shows these lines as drawn between the same two points" $(283)^{4}$. According to Strawson, the falsity of the parallel axiom, which says that there is only one straight line between two points ${ }^{5}$, is unimaginable and inconceivable.

However, one can object to Strawson by claiming that his way of trying to imagine a non-Euclidean space is not the only possible way. First, I will suggest another way of imagining a non-Euclidean sensible world, and then explain the difference between these two ways.

The general theory of relativity predicts that there are regions in our universe where light orbits a black hole. These structures are called "photon spheres." Photon spheres are definitely part of the scientific world, and they are probably part of the sensible world too. It is not possible for us to actually locate ourselves on a photon sphere because it is very unstable and even if it were not, because of the immense gravitational force, we could not survive. But, we can certainly imagine that we are on a photon sphere. On the surface of the photon sphere, a straight line forms a circle around the black hole, because of the curvature of space. As Strawson argues, I cannot imagine a geometrical entity which is both a circle and a straight line at the same time. However, I can imagine this: I look directly forward, and I see my back. I do not try to imagine the shape of the curved space, I just imagine being in a curved space. So, now, cannot I say that a photon sphere is imaginable?

Strawson tries to draw a four-dimensional figure on a three-dimensional canvas, so to speak. What we do in the second approach is to try to imagine the sensible effects of the non-Euclidean space. Being in a non-Euclidean sensible world is

\footnotetext{
4 Strawson (1966).

5 Original formulation of the 5th Axiom by Euclid is as follows "if a straight line falling on two straight lines make the interior angles on the same side less than two right angles, the two straight lines, if produced indefinitely, meet on that side on which are the angles less than the two right angles" (Euclid, Euclid's Elements. Trans. Thomas L. Heath [Michigan: Green Lion Press, 2007]). Strawson uses Hilbert's formulation of the axiom of parallels in David Hilbert, The Foundations of Geometry (Chicago, IL: The Open Court Publishing Company, 1950) 7.
} 
unimaginable in Strawson's approach, but it is imaginable in the second one. So, since non-Euclidean space is imaginable in at least one way, can we say that the sensible world is not necessarily Euclidean?

But, there is a problem with this second approach. What we imagine when we imagine that we are on a photon sphere is that light follows a curved path (curved in the 4th spatial dimension, but straight in the three-dimensional space); however, what we imagine can also be interpreted as light is following a curved path in a Euclidean space. All the effects of a non-Euclidean space on appearances (in Kant's sense) can be interpreted as indications of deformations in the physical entities (objects, light, etc.) and not deformation of the space itself. Of course, we would need much more complicated theories if we try to represent space as Euclidean in our scientific theory, and a theory, like the general theory of relativity, which represents space as non-Euclidean would be much more efficient and convenient.

So, one might argue that when we imagine the effects of curved space, we do not necessarily imagine the curved space. We should be able to imagine not the physical effects of the non-Euclidean space as we would perceive them, but its effects on the geometrical objects like straight lines, planes, etc., as Strawson tries to do. Suppose there are two-dimensional beings living on a flat plane. Suppose that, in their sensible world, there is a phenomenon called "visitor circle." These are circles that appear as a point, then grow, and after a while start to shrink and disappear. Their geometers come up with the three-dimensional Euclidean geometry and their physicists explain the phenomena of visitor circles by theorizing spheres traveling in the three-dimensional space and occasionally passing through their two-dimensional sensible world. Now, the effect of these three-dimensional objects on their two-dimensional world is obviously imaginable for them, and in fact the effect is actual according to our example. But, then, two-dimensional Strawson would ask "are the spheres passing through our world imaginable?" and his answer would be "no." Our Strawson's challenge raises the exact same problem for us. He asks us to imagine the curved straight lines from the perspective of the fourth spatial dimension. In the example of the photon sphere, we imagine within the three-dimensional space, that is why we cannot face the real challenge of imagining a curved space (like the two-dimensional beings imagining a growing and shrinking circle when they try to imagine a passing sphere).

Let us say the two-dimensional beings are living on the surface of a sphere. They need to imagine the third dimension in order to imagine the real space they live in, which is a two-dimensional plane curved in the third dimension. Likewise, in order to imagine a non-Euclidean space properly, we need to imagine a fourth dimension, in which the three-dimensional space we live in is curved. However, as three-dimensional beings, we cannot imagine the fourth dimension. Our minds are not equipped for this task. This is what Strawson's example illustrates. 
The best scientific world of the people living in the two-dimensional world curved in the third dimension might represent the sphere they live on and hence, in the scientific world depicted by their scientists, the space they live in would be three-dimensional. So, it seems, their Kantians would be wrong when they claim that their world is necessarily two-dimensional and flat. But, the question is this: is the third dimension, which becomes a part of their scientific world after their Einstein, also a part of their sensible world?

Sensible world, according to Kant, is partly constructed by a priori mental faculties and pure intuitions. Since the two-dimensional beings live in a twodimensional world and they can never perceive the third dimension, their sensible world is necessarily two-dimensional. The "real" space they are in is three-dimensional, but this is not conceivable for them and not a part of their sensible world. So even though their scientific world is three-dimensional and it explains some phenomena in the sensible world successfully, they are not living in a three-dimensional sensible world, and this is simply because they are two-dimensional beings.

Since the third dimension is not a part of the world of appearances that they live in, but it is real and it affects the appearances in their sensible world, can we say that the third dimension belongs to the world of things in themselves (or the noumenal world) for the two-dimensional beings? But, this would mean that the two-dimensional beings can know something about the noumenal world since their three-dimensional scientific world would represent the noumenal world rather than the sensible world, which is against Kant's views because according to him the noumenal world is unknowable. So, a Kantian cannot argue consistently that Euclidean geometry is about our sensible world and the non-Euclidean geometries are (at least partially) about the noumenal world. It seems, for a Kantian, the only significance of non-Euclidean geometries would be their convenience as mere theoretical tools. In other words, Kantians should endorse scientific instrumentalism when it comes to non-Euclidean geometries and physical theories that rest on non-Euclidean geometries.

So, I argue that non-Euclidean space is inconceivable in one sense and conceivable in another sense. It is inconceivable in the sense that it is not imaginable and it is in principle unperceivable. But it is conceivable in a weaker sense: Non-Euclidean space can be mathematically represented without contradiction. Let us call the first kind of conceivability as "perceptual conceivability" and the second one as "theoretical conceivability." There are examples of the discrepancy between these two conceivabilities. A tesseract, a four-dimensional cube, so to speak, is perceptually inconceivable but theoretically conceivable. Again, an electron in superposition is, arguably, perceptually inconceivable but theoretically conceivable. Consider Poincare's argument against Kant: 
Are they synthetic a priori intuitions, as Kant affirmed? They would then be imposed upon us with such a force that we could not conceive of the contrary proposition, nor could we build upon it a theoretical edifice. There would be no non-Euclidean geometry. ${ }^{6}$

Poincare makes two claims about the non-Euclidean geometries:

(I) If Euclidean geometry is synthetic a priori, then non-Euclidean geometries are inconceivable.

(II) If Euclidean geometry is synthetic a priori, then non-Euclidean geometries cannot be utilized in scientific theories.

It seems to me, Poincare means perceptual conceivability in (I) and theoretical conceivability in (II). I think, then, (I) is true but poses no problem for Kant, since non-Euclidean geometries are perceptually inconceivable. The consequent of (I) is true, so we cannot build a sound Modus Tollens argument with the conclusion that the antecedent of (I) is false. (II), however, is false, since the theoretical world, which refers to non-Euclidean space, includes dimensions that are beyond the sensible world, but our a priori intuitions are only about the sensible world.

As mentioned above, a priori intuitions have two distinct roles for Kant: they are the grounds of the possibility of the sensible world, and also they are the grounds of the possibility of our knowledge (science) of the sensible world. The fact that our best theory about space is non-Euclidean shows that a priori intuitions cannot be the grounds of the possibility of the natural sciences. However, the fact that our scientific world is non-Euclidean does not mean that we should abandon Kant's theory about our a priori intuition of Euclidean empirical space entirely, since our sensible world can still be necessarily Euclidean and a priori intuition of space can still provide an explanation for this metaphysical necessity. Geometry, then, as a science, cannot be synthetic a priori, but the sensible world can still be necessarily Euclidean because of our a priori intuition of space. Therefore, $2^{\prime}$ in the reformulation of the possibility argument, which says "if there are consistent geometries in which the 5th axiom is false and we use at least one of them in our best scientific theory concerning space, then the 5th axiom is possibly false," is not true if we understand the consequent of this conditional statement as "the 5th axiom is possibly false in the sensible world."

\section{Bilkent University}

\footnotetext{
6 Henri Poincare, Science and Hypothesis (New York: The Walter Scott Publishing Co., 1905) 57.
} 